\title{
Non-invasive Neuromodulation in Primary Headaches
}

\author{
Sarah Miller MBBS, MRCP(Neuro), Manjit Matharu FRCP, PhD \\ ${ }^{1}$ Headache Group, Institute of Neurology and The National Hospital for Neurology \\ and Neurosurgery, Queen Square, London, UK
}

\section{Corresponding Author:}

Dr Sarah Miller

Clinical Research Fellow

Headache Group, Institute of Neurology and The National Hospital for Neurology and Neurosurgery, Queen Square, London WC1N 3BG

Email: sarah.miller.12@ucl.ac.uk

Tel: +447595900535

Fax: +447092120797

Keywords: Trigeminal autonomic cephalalgia; Migraine; Neurostimulation; Vagal nerve stimulation; Supraorbital nerve stimulation; Transcranial magnetic stimulation 


\begin{abstract}
Purpose of review: There is growing interest in neuromodulation for primary headache conditions. Invasive modalities such as occipital nerve stimulation, deep brain stimulation and sphenopalatine ganglion stimulation are reserved for the most severe and intractable patients. Non-invasive options such as vagal nerve stimulation (nVNS), supraorbital nerve stimulation (nSONS) and transcranial magnetic nerve stimulation (TMS) have all emerged as potentially useful headache treatments. This review examines the evidence base for non-invasive neuromodulation in trigeminal autonomic cephalalgias and migraine.
\end{abstract}

Recent findings: Although a number of open-label series of non-invasive neuromodulation devices have been published there is very little controlled evidence for their use in any headache condition. Open-label evidence suggests that nVNS may have a role in the prophylactic treatment of cluster headache and there is limited evidence to suggest it may be useful in the acute treatment of cluster and potentially migraine attacks. There is limited controlled evidence to suggest a role for nSONS in the prophylactic treatment of episodic migraine but there is no evidence to support its use in cluster headache. TMS may be efficacious in the acute treatment of episodic migraine has no controlled evidence to support its use as a preventative in any headache condition.

Summary: Non-invasive neuromodulation techniques are an attractive treatment option with excellent safety profiles but their use is not yet supported by high-quality randomized controlled trials. 


\section{INTRODUCTION}

Primary headache conditions, especially migraine, are a cause of significant disability and economic burden worldwide. Although the treatment options for primary headaches have progressed with time, there is still a major issue with the efficacy, availability, adverse event and tolerability profiles of current pharmaceutical agents. It is estimated that under $25 \%$ of chronic migraine patients continue taking oral preventative agents for more than 12 months due to lack of efficacy or tolerability issues (1). In cluster headache $(\mathrm{CH})$, besides the above issues with preventative agents, there are major limitations to acute treatments also. For example, subcutaneous sumatriptan is expensive and can only be used twice daily even in those with more frequent attacks and the triptans are also contraindicated in those with heart disease or uncontrolled blood pressure. Oxygen, the only other evidence based acute treatment for $\mathrm{CH}$, may be effective in the early stages of attack but is often associated with attack recurrence and is not as portable as patients would like.

Neuromodulation techniques have evolved in order to combat some of these issues. Neuromodulation works by using electricity or magnetic impulses to manipulate the neurotransmitter systems such as serotonin, glutamine and dopamine important in the pain neuromatrix (Figure 1). Invasive neuromodulation therapies such as occipital nerve stimulation (ONS), deep brain stimulation (DBS) and sphenopalatine ganglion stimulation (SPGS) are all limited for use in highly refractory patients due to their invasive nature and cost (2). However, the recent development of non-invasive neuromodulation therapies for primary headache conditions offer a non-surgical approach which may be more accessible and acceptable for patients. Currently available non-invasive neuromodulation technologies include supraorbital transcutaneous stimulation, non-invasive vagal nerve stimulation and transcranial magnetic stimulation. With regards to the trigeminal autonomic cephalalgias 
(TACs) the evidence is limited to non-invasive vagal nerve stimulation and so this review will focus on this technology but the potential use of the devices in other primary headache conditions will be discussed (Table 1).

\section{NON-INVASIVE VAGAL NERVE STIMULATION (nVNS)}

The vagus nerve is a mixed motor and sensory nerve involved in autonomic, respiratory cardiovascular, gastrointestinal and pain systems. Efferent fibres of the vagal nerve arise from the nucleus ambiguus and dorsal motor nucleus of the vagal nerve. Afferent fibres terminate on the area postrema, the spinal nucleus of the trigeminal nerve and the nucleus of the solitary tract. There is anatomical evidence of the role the vagus nerve may play in regulating trigeminal pain. The nucleus of the solitary tract has been shown to receive nociceptive afferents from the dura (3). Studies in rats have also shown that vagus nerve stimulation reduces pain and allodynia in the trigeminal distribution, an observation proposed to be secondary to an ascending antinoceceptive effect of the vagus nerve on the second order neurons of the spinothalamic and spinoreticular tracts (4). There is also evidence that vagal nerve stimulation reduces glutamate levels and firing rate in the spinal trigeminal nucleus. Recent neuroimaging studies have suggested that chronic vagus nerve stimulation inhibits activation in the thalamus, limbic system, dorsal pons, locus coerulus and nucleus of the solitary tract, all structures previously identified as parts of the pain matrix in headache (5) (Figure 1).

Following reports of improvement of migraine in patients with epilepsy receiving invasive vagal nerve stimulation, VNS devices were implanted in a limited number of refractory headache patients with positive case reports in migraine and cluster headaches $(6,7)$. Recently, a portable transcutaneous non-invasive vagal nerve stimulator has been developed 
$\left(\right.$ GammaCore $\left.^{\circledR}\right)$ that stimulates the cervical portion of the vagus nerve. The device is placed on the neck and then a mild electrical current is applied to the skin. The treatment has been trialled for both cluster headache and migraine.

\section{$\underline{\text { nVNS in cluster headache }}$}

\section{Preventative treatment of cluster headache (Table 1)}

The initial potential of $\mathrm{nVNS}$ in the preventative treatment of $\mathrm{CH}$ was explored in an audit by Nesbitt et al. in 2015 (8). Of 19 patients included, 11 suffered chronic $\mathrm{CH}(\mathrm{CCH})$ and eight were episodic $(\mathrm{ECH})$. Seven patients were reported as refractory to drug treatments (9). The preventative regime consisted of two to three stimulations (doses) in the morning and late afternoon with stimulation delivered on the side of the neck ipsilateral to the cluster attacks. Fifteen patients reported an improvement after one year, with a mean estimated improvement of $48 \% \pm 9 \%$. There were no reports of any serious adverse events although two patients experienced side-shifting of attacks. There were problems with this study including the lack of placebo, the lack of objectively measured outcomes and the ability of patients to continue and change their preventative medications alongside nVNS. Regards the last point, one patient started methysergide, one increased verapamil dosage and one was treated with high dose steroids during the study period. However, it did suggest a potential role for nVNS for the preventative treatment of $\mathrm{CH}$.

A larger prospective, open-label randomised study of nVNS in $\mathrm{CCH}$ (the prevention and acute treatment of chronic cluster headache or PREVA trial) was then conducted by Gaul et al. in 2015 (10). This multicentre trial of 119 patients with CCH compared adjunctive prophylactic nVNS plus standard care $(n=48)$ to standard care alone (control) $(n=49)$ for four weeks followed by a four-week extension of standard care and nVNS. The nVNS preventative regime 
consisted of three 2-minute stimulations five minutes apart administered twice daily to the right side of the neck. The first dose was administered on waking and the second dose seven to 10hours later. Changes in standard care preventative medicines were not allowed. The intention to treat population on which analysis was performed consisted of $45 \mathrm{nVNS}$ and 48 control patients. Adherence of $>80 \%$ to nVNS treatment was observed in $64.4 \%$ of patients in those initially randomised to nVNS from the start and $50 \%$ in those receiving nVNS during the extension phase only. In the randomised phase, those receiving nVNS had 3.9 (95\% confidence interval 0.5,7.2; $\mathrm{p}=0.002$ ) fewer attacks a week than controls. In the longer-term, those using nVNS throughout the extension period reported an additional reduction of two $\mathrm{CH}$ attacks a week suggestive of increased benefit with continued use. In those adding nVNS in the extension phase, individuals reported a reduction of $3.3 \mathrm{CH}$ attacks a week compared to standard care alone $(15.7$ vs. $12.4 ; \mathrm{p}<0.001)$. During the randomised phase, the $\geq 50 \%$ response rate was significantly higher in the nVNS group (40\%) compared to the control group $(8.3 \%)$. Data suggested that those continuing to use nVNS continued to respond throughout the extension phase. Although no serious adverse events were related to the use of nVNS, seven individuals did discontinue due to side effects (feeling hot, malaise, haematoma after planned surgery and depression). At the study end, $65 \%$ recommended the nVNS to others and over $50 \%$ were satisfied with their response to nVNS. Although this study did not have a placebo group, it did consist of $\mathrm{CCH}$ patients alone and it was felt such patients are less likely to undergo periods of spontaneous remission than those with ECH.

The cost-effectiveness of nVNS for preventative treatment of $\mathrm{CCH}$ was recently analysed using the PREVA data (11). Using a model based on German health insurance costs the yearly cost of nVNS and standard care was $€ 7096.69$ with quality-adjusted life-years (QALY) of 0.607 compared to $€ 7511.35$ with QUALY of 0.522 for standard care alone. Abortive medication costs were $23 \%$ lower in the nVNS group than standard care. Similar analysis applied to a UK 
perspective found similar results. On the basis of this data, nVNS appears to generate greater benefit for less economic cost then standard care alone. Given that the model did not include possible savings from changes in preventative medications, medical appointments or indirect costs (e.g. ability to work), the benefits of nVNS have likely been underestimated in this study. Taken together, this open-label evidence appears to suggest that adjunctive prophylactic nVNS is a potentially effective, well-tolerated and cost-effective treatment for the prevention of $\mathrm{CH}$.

\section{Acute treatment of cluster headache attacks (Table 1)}

The audit from Nesbitt et al. reported that patients could terminate attacks within 11 minutes \pm 1 minute of device application in the acute setting (8). The use of nVNS as an acute treatment allowed ten patients to reduce their oxygen usage by an estimated $55 \%$, four to stop triptans completely and a further nine to reduce triptan intake by $48 \%$. In the study by Gaul et al. patients were given the option to treat three attacks daily in addition to their preventative doses with instructions to take normal rescue medication in the attack was not terminated within 15 minutes (10). During the randomised phase $93.8 \%$ used the device as an acute treatment at least once. However, no evidence was found of a reduction in attack duration or pain intensity with acute use.

On the basis of available open-label data there is little evidence to support the use of nVNS for the acute treatment of $\mathrm{CH}$.

nVNS in other TACs (Table 1)

There are no published data on the use of nVNS in any of the other TACs. Our own clinical observations, on having used it in a handful of paroxysmal hemicrania $(\mathrm{PH})$, hemicrania 
continua (HC) and short lasting unilateral neuralgiform headache attacks (SUNHA) are that it may be useful in some of these conditions as a preventative treatment (PH, HC and SUNHA) or when used as an acute treatment $(\mathrm{PH})$. However, further studies are needed to explore the efficacy and safety of nVNS in these rare headache disorders

\section{$\underline{\text { nVNS in migraine }}$}

\section{Preventative treatment of migraine (Table 1)}

As in $\mathrm{CH}$, there is no controlled evidence to support the use of nVNS in migraine. However, two open-label series have investigated its potential role. One small study from Magis et al. (available in abstract form only) reported outcomes of 12 patients with migraine treated with nVNS (12). Ten patients discontinued the treatment because of lack of efficacy or adverse effects. A recent double-blinded, sham-controlled pilot study of nVNS in the preventative treatment of CM examined 59 participants randomised to $\mathrm{nVNS}$ or sham for two months before receiving open-label nVNS. Treatment consisted of two doses delivered three times a day. At the end of the blinded phase there had been a 1.9 day reduction in headache days in the active compared to 0.2 day change in the sham group. After six months, those receiving nVNS from outset had a mean change of -2.5 headache days a month suggesting that longer use is needed to optimise effect. A study of the preventative role of $n V N S$ in episodic migraine is ongoing.

\section{Acute treatment of migraine (Table 1)}

Three open-label studies have examined the use of nVNS in acute migraine attacks. The first from Goadsby et al. reported on 27 patients with and without aura who were treated with two 90-second doses at 15-minute intervals during an attack (13). For all moderate to severe treated 
attacks, the pain-free rate at two hours was $22 \%$ with $43 \%$ reporting significant reductions in pain scores. These results are comparable to naproxen 500mg. Side effects were mild and infrequent. Another study (available in abstract form only) of 22 patients with 79 treated attacks showed $\geq 50 \%$ reduction in pain scores at two hours in $46 \%$ of patients (12). The final study included 48 patients with CM or high frequency episodic migraine treating 131 attacks over a two-week period (14). Treatment consisted of two 120-second doses at three-minute intervals. At two hours, $64.6 \%$ reported $\geq 50 \%$ reduction in pain scores and $39.6 \%$ were pain free. Treatment was well tolerated with no major side effects. nVNS may be effective as an acute treatment for migraine but larger controlled studies are needed.

\section{SUPRAORBITAL NERVE STIMULATION}

The supraorbital nerve is derived from the first branch of the trigeminal nerve (V1). The trigeminal nerve is responsible for the relay of nociceptive information from intracranial structures such as the dura and large blood vessels. C-fibres innervating these structures pass through the trigeminal ganglion and into the trigeminal nucleus caudalis (TNC) in the pons. The TNC extends caudally and connects with the C1-C3 levels of the spinal cord and rostrally projects to the thalamus, the superior salivatory nucleus and hypothalamus (Figure 1). This pathway is crucial in the pathophysiology of headache pain and is explored in numerous articles (15). Recent preliminary results from an FDG-PET study in episodic migraine following treatment with non-invasive supraorbital nerve stimulation (nSONS) showed a normalisation of orbitofrontal hypometabolism, suggesting the treatment has its effect via a neuromodulatory effect involving the limbic system and areas of the pain matrix.

Case reports of supraorbital nerve stimulator percutaneous implants, often alongside occipital nerve stimulation, have suggested a possible beneficial effect in migraine, cluster headache and 
short lasting-unilateral neuralgiform headache attacks with conjunctival injection and tearing (16). However, invasive nerve stimulation is reserved for refractory patients only. A transcutanoues supraorbital nerve stimulator, the Cefaly ${ }^{\circledR}$ device has now been developed to provide nSONS and has been granted American Food and Drug Administration (FDA) approval in episodic migraine.

\section{$\underline{\text { nSONS in the trigeminal autonomic cephalalgias }}$}

Currently there is no evidence that nSONS using the Cefaly ${ }^{\circledR}$ device has any role in either the acute or preventative treatment of TACs (Table 1). Our own clinical observations are that the device is not useful in either the preventative or acute treatment of TACs. However, given the data on invasive SONS in CCH well planned controlled trials are needed.

\section{$\underline{\text { nSONS in migraine }}$}

Preventative treatment of episodic migraine (Table 1)

Pilot trials in 10 patients suggested efficacy and safety of nSONS in episodic migraine (17). This led to a randomised sham-controlled multicentre study conducted between 2009-2011. The PREvention of Migraine using Cefaly (PREMICE) study included 67 patients with at least two migraine attacks a month who were randomised to either verum or sham nSONS for a three-month treatment period (18). Treatment consisted of one 20-minute session daily for both groups. After three-months, migraine days decreased significantly in the active group (2.06 days; $\mathrm{p}=0.023)$ but not in the sham group $(0.32 ; \mathrm{p}=0.608)$, however, the difference between migraine day reduction between groups did not reach statistical significance. The $50 \%$ responder rate was $38.1 \%$ in the active and $12.1 \%$ in the sham group $(\mathrm{p}=0.023)$. 
Additional statistical analysis conducted after this publication was performed which discovered that those with more frequent migraine derived more benefit from the nSONS (19). No adverse events were recorded in either group.

A post marketing study of 2313 subjects using nSONS as a preventative agent reported $53 \%$ $(n=1236)$ to be "satisfied" with treatment in that they continued to use the device post the 40day free trial period (20). However, of those returning the device, 48 subjects had failed to even turn the device on and 157 had not used the device for more than 60 minutes. In total, $40 \%$ of those returning the device $(n=431)$ had failed to comply with treatment and it has been calculated that the true non-responder rate could be as low as $18.6 \%$. No serious adverse events were reported.

Acute treatment of episodic migraine (Table 1)

There is no evidence to support the use of nSONS in the acute treatment of migraine. The original pilot study mentioned above also treated 30 attacks with nSONS with total relief in just $13 \%$ and partial relief with additional medication in $45 \%$.

\section{TRANSCRANIAL MAGNETIC STIMULATION}

The brain of migraine sufferers is thought to be hyperexcitable, a theory supported by transcranial magnetic stimulation studies (21). This leads to a lowered threshold for cortical spreading depression, a process linked to the generation of migraine aura and pain. Transcranial magnetic stimulation (TMS) applies a brief single magnetic pulse to the scalp and underlying cortex. This pulse induces electric field potentials in the cortex, alternating neurotransmitter levels and disrupting cortical spreading depression (Figure 1). Animal studies 
have shown the TMS inhibits cortical spreading depression. It was extrapolated that in acute migraine attacks, TMS could terminate aura and reduce pain levels. A portable TMS device (SpringTMS) was thus developed as a migraine treatment.

\section{$\underline{\text { TMS in trigeminal autonomic cephalalgias }}$}

There is no evidence to support the use of TMS in TACSs and given that the pathophysiology of TACs does not include cortical spreading depression it is unlikely to be a focus of investigation in the near future.

\section{$\underline{\text { TMS in migraine }}$}

Preventative treatment of episodic migraine (Table 1)

There is no controlled evidence to support the use of TMS in the preventative treatment of migraine.

Acute treatment of episodic migraine with and without aura (Table 1)

A single sham-controlled study on the use of TMS in the acute treatment of migraine with aura reported a pain-free at $2 \mathrm{~h}$ response rate of $39 \%$ compared to $22 \%$ in sham treated patients, a difference that was maintained at $24 \mathrm{~h}$ (22). Open-label post-marketing surveys conducted on 462 subjects using TMS as an acute treatment for migraine with and without aura reported full 3-month follow-up data on only 190 patients with a discontinuation rate of 55\% (23). The group have not provided complete data on their responder rates but stated that $62 \%$ reported 
"some reduction" in migraine pain. On this evidence, TMS may have a role in the acute treatment of migraine but more data is obviously needed.

\section{CONCLUSION}

There is a major unmet need in the currently available treatment options for patients with primary headache conditions, including those with trigeminal autonomic cephalalgias. Noninvasive neuro-modulation techniques offer potential efficacy combined with highly favourable adverse effect profiles. There is also some suggestion that their cost-effectiveness will also prove favourable compared to standard treatments. However, despite some promising initial open-label data, caution has to be used in deciding when to use these treatments. At present, there are no high quality double-blinded randomised controlled trials on the use of any non-invasive neuromodulation techniques for primary headache conditions and these will be needed before decisions can be made on the true role of non-invasive neuromodulation. At present, these devices should be reserved for those with medication tolerance issues or those proving intractable to standard treatments. Clinicians have a responsibility to explain the limited evidence base and possible limitations of these treatment modalities to patients. However, if robust evidence on non-invasive neuromodulation techniques can be obtained, these treatments are likely to become a valuable part of our management options. 


\section{REFERENCES:}

1. Hepp Z, Bloudek LM, Varon SF. Systematic review of migraine prophylaxis adherence and persistence. J Manag Care Pharm. 2014;20(1):22-33.

2. $\bullet$ Martelletti P, Jensen RH, Antal A, Arcioni R, Brighina F, de Tommaso M, et al. Neuromodulation of chronic headaches: position statement from the European Headache Federation. J Headache Pain. 2013;14(1):86. EFNS guidelines on the use of neuromodulation techniques with guidance on when to use each modality

3. Goadsby PJ, Hoskin KL. The distribution of trigeminovascular afferents in the nonhuman primate brain Macaca nemestrina: a c-fos immunocytochemical study. J Anat. 1997;190 ( Pt 3):367-75.

4. Bossut DF, Maixner W. Effects of cardiac vagal afferent electrostimulation on the responses of trigeminal and trigeminothalamic neurons to noxious orofacial stimulation. Pain. 1996;65(1):101-9.

5. Kraus T, Kiess O, Hosl K, Terekhin P, Kornhuber J, Forster C. CNS BOLD fMRI effects of sham-controlled transcutaneous electrical nerve stimulation in the left outer auditory canal - a pilot study. Brain Stimul. 2013;6(5):798-804.

6. Sadler RM, Purdy RA, Rahey S. Vagal nerve stimulation aborts migraine in patient with intractable epilepsy. Cephalalgia. 2002;22(6):482-4.

7. Mauskop A. Vagus nerve stimulation relieves chronic refractory migraine and cluster headaches. Cephalalgia. 2005;25(2):82-6.

8. Nesbitt AD, Marin JC, Tompkins E, Ruttledge MH, Goadsby PJ. Initial use of a novel noninvasive vagus nerve stimulator for cluster headache treatment. Neurology. 2015.

9. Goadsby PJ, Schoenen J, Ferrari MD, Silberstein SD, Dodick D. Towards a definition of intractable headache for use in clinical practice and trials. Cephalalgia. 2006;26(9):1168-70. 
10. $\bullet$ Gaul C, Diener HC, Silver N, Magis D, Reuter U, Andersson A, et al. Non-invasive vagus nerve stimulation for PREVention and Acute treatment of chronic cluster headache (PREVA): A randomised controlled study. Cephalalgia. 2016;36(6):534-46. The only randomised controlled study of non-invasive neuromodulation showing a potential use of $n$ VNS in chronic cluster headache

11. -Morris J, Straube A, Diener HC, Ahmed F, Silver N, Walker S, et al. Costeffectiveness analysis of non-invasive vagus nerve stimulation for the treatment of chronic cluster headache. J Headache Pain. 2016;17:43. Paper examing the costeffectiveness of $n$ VNS for CCH compared to standard treatment, the only paper to look at cost-benefit of non-invasive neuromodulation

12. Magis D, Gerard P, Schoenen J. Abstracts of the European Headache and Migraine Trust International Congress, September 20-23, 2012, London, United Kingdom. J Headache Pain. 2013;14 Suppl 1:198.

13. Goadsby P, Grosberg B, Mauskop A, Cady R, Simmons K. Effect of noninvasive vagus nerve stimulation on acute migraine: An open-label pilot study. Cephalalgia. 2014;34(12):986-93.

14. Barbanti P, Grazzi L, Egeo G, Padovan AM, Liebler E, Bussone G. Non-invasive vagus nerve stimulation for acute treatment of high-frequency and chronic migraine: an openlabel study. J Headache Pain. 2015;16:61.

15. Bartsch T, Goadsby PJ. The trigeminocervical complex and migraine: current concepts and synthesis. Current pain and headache reports. 2003;7(5):371-6.

16. Goldberg SW, Nahas SJ. Supratrochlear and Supraorbital Nerve Stimulation for Chronic Headache: a Review. Current pain and headache reports. 2015;19(7):26.

17. Gerardy PY, Fabry D, Fumal A, Schoenen J. A pilot study on supra-orbital surface electrotherapy in migraine. Cephalalagia. 2009;29:101-78. 
18. Schoenen J, Vandersmissen B, Jeangette S, Herroelen L, Vandenheede M, Gerard P, et al. Migraine prevention with a supraorbital transcutaneous stimulator: a randomized controlled trial. Neurology. 2013;80(8):697-704. Outcome data from the PREMICE study on migraine prevention with SONS

19. Schoenen JE. Migraine prevention with a supraorbital transcutaneous stimulator: A randomized controlled trial. Neurology. 2016;86(2):201-2. 20. Magis D, Sava S, d'Elia TS, Baschi R, Schoenen J. Safety and patients' satisfaction of transcutaneous supraorbital neurostimulation (tSNS) with the Cefaly(R) device in headache treatment: a survey of 2,313 headache sufferers in the general population. J Headache Pain. 2013;14:95.

21. Lipton RB, Pearlman SH. Transcranial magnetic simulation in the treatment of migraine. Neurotherapeutics. 2010;7(2):204-12.

22. Lipton RB, Dodick DW, Silberstein SD, Saper JR, Aurora SK, Pearlman SH, et al. Single-pulse transcranial magnetic stimulation for acute treatment of migraine with aura: a randomised, double-blind, parallel-group, sham-controlled trial. Lancet Neurol. 2010;9(4):373-80.

23. Bhola R, Kinsella E, Giffin N, Lipscombe S, Ahmed F, Weatherall M, et al. Singlepulse transcranial magnetic stimulation (sTMS) for the acute treatment of migraine: evaluation of outcome data for the UK post market pilot program. J Headache Pain. 2015;16:51. 